\title{
Plasmablastic lymphoma presenting as a soft tissue mass associated with a chronic wound in an immunocompetent patient
}

\author{
Salwa Khedr ${ }^{1}$. Chibuike Leonard Enwereuzo ${ }^{1}$ (D) Sharon Secola ${ }^{1} \cdot$ Nicholas Mai ${ }^{1} \cdot$ Patricia Miron ${ }^{1}$. \\ Lloyd Hutchinson ${ }^{1} \cdot$ Benjamin Chen ${ }^{1}$
}

Received: 1 May 2020 / Accepted: 2 July 2020 / Published online: 11 July 2020

(C) Springer-Verlag GmbH Germany, part of Springer Nature 2020

\begin{abstract}
Plasmablastic lymphoma (PBL) is a rare aggressive type of non-Hodgkin lymphoma that primarily affects human immunodeficiency virus (HIV)-positive patients but can arise in other immunocompromised states such as transplant recipients, as well as in immunocompetent patients. The neoplastic cells express an immunophenotype that resembles plasma cells, in that they usually do not express CD20 (or may be weakly positive) but do express markers of plasmacytic differentiation such as CD38, CD138, or MUM1/IRF4. These tumors generally have high mitotic activity, evidenced by a high Ki-67 proliferation index. Epstein-Barr virus $(\mathrm{EBV})$ is frequently detected within the tumor and is thought to have a pathogenic role in the development of PBL. The earliest descriptions of PBL were as oral cavity lesions. PBL most frequently present as a mass in extranodal regions of the head and neck. Presentation as a soft tissue mass is very uncommon. In this article, we present an immunocompetent 50-year-old male patient who presented with a soft tissue left heel mass in the setting of chronic ulceration, subsequently diagnosed as PBL.
\end{abstract}

Keywords Plasmablastic lymphoma $\cdot$ Non-Hodgkin lymphoma $\cdot$ Immunocompetent

\section{Introduction}

Plasmablastic lymphoma (PBL) is currently a distinct diagnostic entity in the WHO classification of hematologic neoplams and was first described in 1997 by Delecluse, et al. [1]. It is associated with Epstein-Barr virus (EBV) infection and immunodeficiency states especially HIV-infected individuals with a predilection for the oral cavity. PBLs have also been described in HIV-negative and immunocompetent individuals [2-4].

PBL is composed of B cells that have undergone preterminal differentiation to plasma cells $[5,6]$. The morphology varies according to the site of involvement. In its most common site, the oral cavity, the neoplastic population consists of immunoblastic cells with abundant cytoplasm, vesicular chromatin, and centrally placed nucleoli [7]. In lymph nodes and other extranodal sites, the neoplastic cells show a greater degree of plasmacytic differentiation. Confluent areas of

Chibuike Leonard Enwereuzo

chibuikeenwereuzo@gmail.com

1 Department of Pathology, University of Massachusetts Medical School, 1 Innovation Dr \#2, Worcester, MA 01605, USA necrosis and a starry sky background with tingible body macrophages have been described [8]. The differential diagnosis includes plasma cell myeloma with blastic morphology, extracavitary primary effusion lymphoma, and ALK-positive large B cell lymphoma. The characteristic immunophenotype of PBL is typically positive for CD138, CD38, and MUM1 and negative for CD20, PAX5, and CD45. CD79a is often expressed. Cases have been described with positive staining for CD4, CD10, CD30, and/or CD56 $[8,9]$.

The largest meta-analysis of PBLs to date (277 cases mostly involving unusual case reports) notes that half of the individuals were positive for HIV and another $14 \%$ had transplant related immunosuppression. There were a substantial percentage of the HIV negative patients who were immunocompetent. In immounocompetent individuals, nodal involvement was predominant. There have been rare reports of EBV negative PBLs, and those cases were associated with poorer prognosis compared with individuals with EBV positive PBLs $[2,3]$.

\section{Case report}

Our patient is a 50-year-old male with no significant past medical history who sustained a fall 3 years ago, resulting in 
a left calcaneal comminuted fracture. The patient underwent an open reduction and internal fixation with placement of a rod. The surgical wound, located on the medial side of the calcaneus, failed to heal and increased in size over 1 year with unintentional 25-pound weight loss. A punch biopsy of the wound showed sheets of malignant-appearing epithelioid cells with prominent nucleoli located in the papillary dermis with areas of necrosis, intermixed with inflammatory cells (Fig. 1). There was no epidermal involvement. Immunohistochemical studies revealed that the tumor cells were negative for CD3, CD20, CD 45, P63, EMA, CD 31, CD34, CD99, synaptophysin, chromogranin, desmin, Myo-D1, WT1, OCT3/4, Sall-4, INI-1 (intact nuclear expression), CD68, lysozyme, and myeloperoxidase. A particular line of differentiation was not definitively identified. At that time, the differential diagnosis included a poorly differentiated carcinoma and a high-grade sarcoma. A hematopoietic lesion was considered unlikely given the presentation and CD45 negativity.

Due to the nature and the location of the mass, surgical management was considered. Local excision was not deemed possible, and an amputation was determined to be the best course of therapy from an oncologic and functional standpoint. A left below the knee amputation was performed.

A left below the knee amputation was performed (Fig. 2). Gross examination of the specimen revealed an $8 \times 7.5 \times 4 \mathrm{~cm}$ ulcerating lesion on the dorsum of the left heel exposing underlying bone. Cut surface of the ulcerated lesion was red, fleshy, and soft. The underlying bone appeared to be grossly involved. On microscopy, the lesion had better morphology compared with the previous biopsy and the tumor cells appeared large, pleomorphic, and plasmacytoid with eccentric irregular nuclei, coarse chromatin, prominent nucleoli, and eosinophilic cytoplasm with extensive necrosis (Fig. 3). Immunohistochemical studies showed focal staining for CD45 and positive staining for CD79a, prompting suspicion for a lymphoproliferative neoplasm. CD20 and CD3 were negative in neoplastic cells (Fig. 4). Further studies showed that the neoplasm was negative for PAX5 and CD30 and positive for MUM1, CD138 (multifocal), and CD38 (weak,

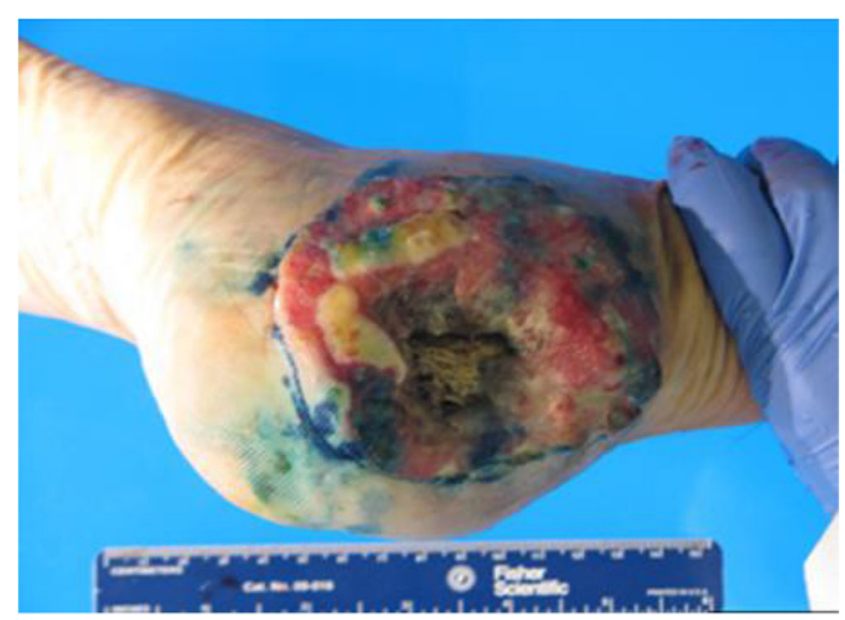

Fig. 2 Gross image of lesion in the amputated foot

multifocal) (Fig. 5). C-MYC was overexpressed, estimated to be $80-90 \%$ of tumor cells, and the Ki67 proliferation index was approximately $90 \%$. Additional negative stains included CD5, myeloperoxidase, CD34, CD68, ALK1, and CD43 which were performed to rule out other lymphoproliferative neoplasms including anaplastic large cell lymphoma, ALKpositive large B cell lymphoma, and myeloid sarcoma. This phenotype points to a neoplasm derived from a terminally differentiated B cell, as evident by the negativity for CD20 and PAX5 and positivity for CD138, CD38, and MUM1. At this point, the differential diagnosis included a plasma cell myeloma and a PBL. EBER in situ hybridization was positive (Fig. 6), which is highly associated with plasmablastic lymphoma, and CD56, which is associated with plasma cell myeloma, was negative. There was kappa light chain restriction, as evident by kappa and lambda in situ hybridization studies (Fig. 6). Additionally, the negative serum protein electrophoresis and absence of lytic bone lesions on imaging studies helped to rule out plasma cell myeloma.

Chromosome analysis of seven metaphase cells showed a complex karyotype described by the following composite karyotype: 33-47,-X,-Y,t(1;13)(q12;p11.2),+ $6, \mathrm{t}(8 ; 14)(\mathrm{q} 24 ; \mathrm{q} 32), \operatorname{der}(9) \mathrm{t}(1 ; 9)(\mathrm{q} 12 ; \mathrm{p} 24)$, add $[10]$

Fig. 1 Skin biopsy showing a low power view with a diffuse dermal infiltrate of a neoplastic population of cells (a) and a high power view displaying large pleomorphic cells with open nuclear chromatin and prominent nucleoli (b)
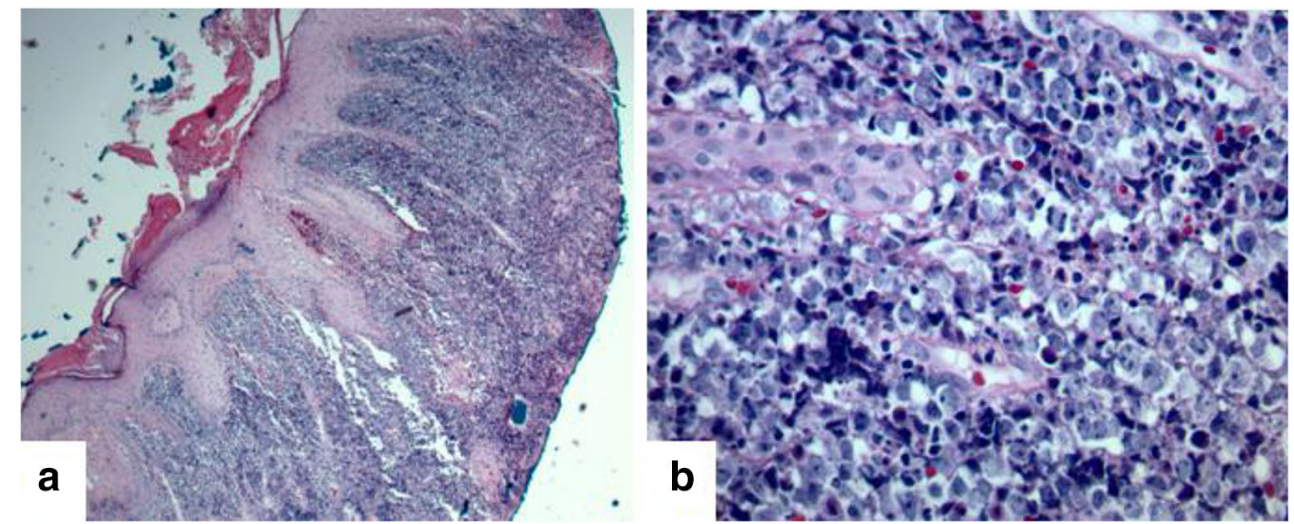
Fig. 3 Histopathologic images of the amputation specimen shows dermal involvement (a) by a diffuse neoplastic population of plasmacytoid cells with

pleomorphic nuclei (b, c, and d). Tumor invasion into the subcutaneous tissue (e). Tumor and bone necrosis (f)

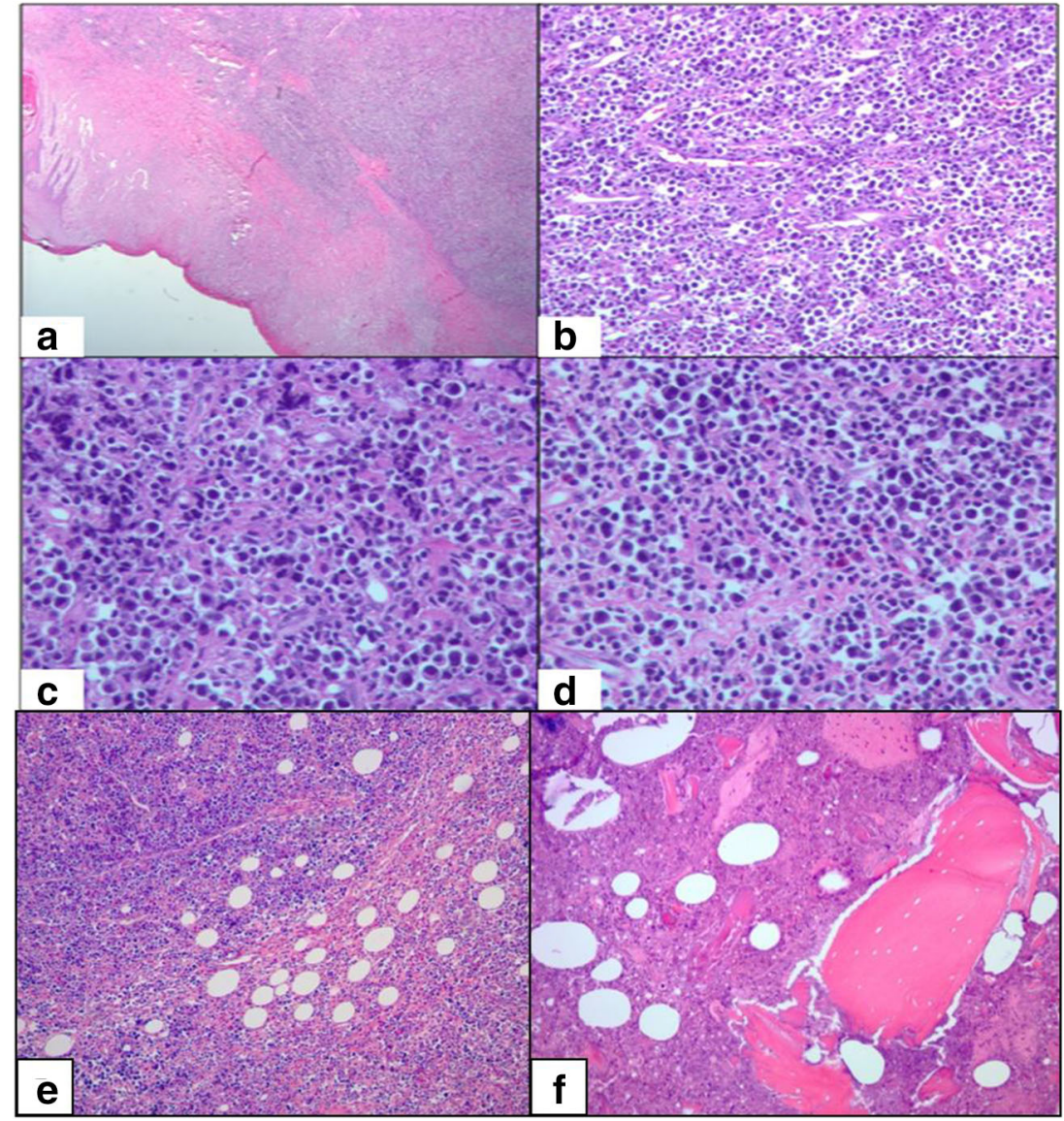

formed from the $t(8 ; 14)$. Interphase fluorescent in situ hybridization (FISH) with a MYC-IGH probe set was consistent with the karyotype, with an abnormal pattern in 21/50 cells consistent with a MYC-IGH translocation
Fig. 4 The initial battery of immunohistochemical stains shows that the neoplastic cells are negative for $\mathrm{CD} 20$ and $\mathrm{CD} 3$ with some intervening smaller $\mathrm{T}$ cells. The neoplastic population shows weak positive subset staining for $\mathrm{CD} 45$ and positive staining for CD79a
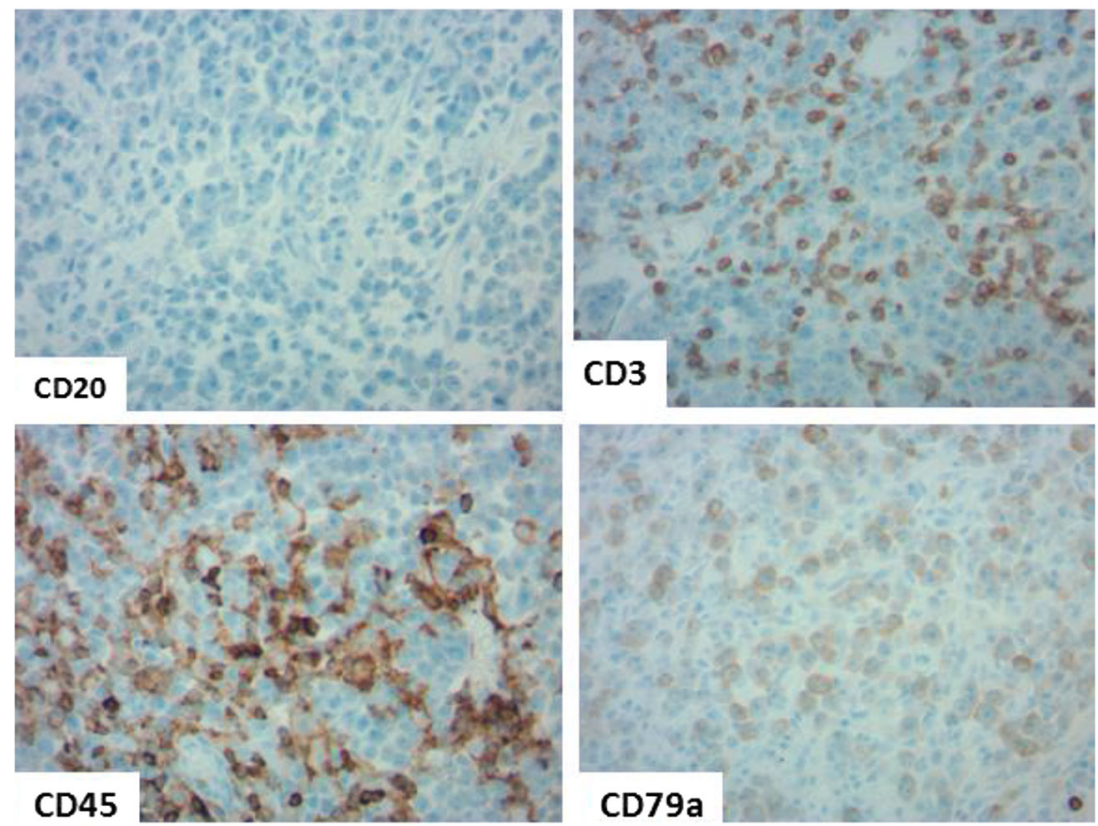
Fig. 5 The tumor cells are positive for MUM1, CD138 (multifocal), and CD38 (weak, multifocal)
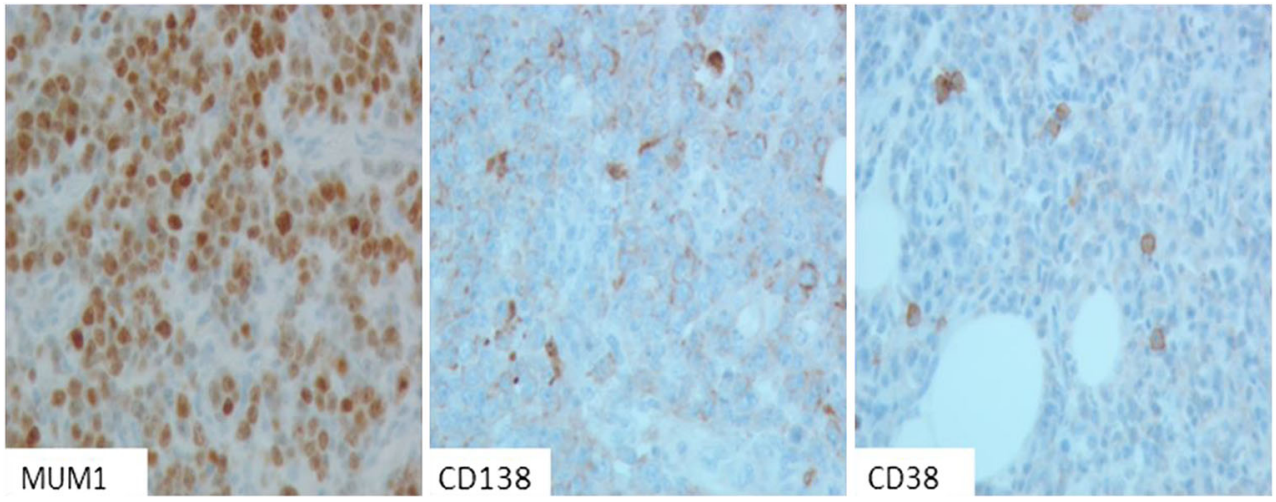

and an extra copy of a MYC-IGH fusion. B cell clonality studies showed immunoglobulin heavy chain gene and kappa light chain gene clonal rearrangement.

In conjunction with the clinical history, morphology, immunohistochemistry, and ancillary tests, the results were most consistent with a PBL. Diffuse large B cell lymphoma associated with chronic inflammation was considered in the differential diagnosis, but those tend to have mature B cell phenotype with only occasional plasmacytic differentiation, and a proliferative index of $90 \%$ is unusual for this entity. Additional testing with markers of immune checkpoints showed positive staining for PDL1 and TIM3, but negative staining for LAG3 and PD1 (Fig. 7). This pattern of expression of immune checkpoint protein further supports classification as plasmablastic lymphoma. A retroviral screening for HIV 1 and 2 was done and found to be negative.

\section{Materials and methods}

Four-micron-thick sections of $10 \%$ neutral buffered formalinfixed paraffin-embedded (FFPE) sections were stained with hematoxylin and eosin for histologic examination. Immunohistochemical studies were done on the Ventana automated immunostainer (BenchMark ULTRA, Ventana Medical Systems). In situ hybridization was done with an EBER 1 DNP probe in combination with the Ventana ISH iVIEWblue Plus Detection system. Cytogenetic was performed by analysis of GTG-banded chromosomes on seven metaphase cells. Interphase FISH with a MYC-IGH dual fusion/dual color probe set (Abbott Molecular) was performed on 50 interphase cells from a direct preparation of fresh tissue. Multiplex polymerase chain reaction (PCR) for immunoglobulin (IG) gene rearrangement was performed using standard PCR protocol [8-10].

\section{Discussion}

Only a few PBLs have been reported in the soft tissue in HIV negative individuals [2]. We believe that this patient was immunocompetent; however, due to his chronic wound infection, this chronic inflammatory stimulation could have contributed to development of the PBL. Chronic inflammation has been associated with development of diffuse large B cell lymphomas. A common scenario is pyothorax associated lymphoma that develops in long-standing chronic pyothorax and has morphologic features of diffuse large B cell lymphoma. These lymphomas associated with chronic inflammation are usually EBV positive but tend to have complete B cell immunophenotype. It has been postulated that the pathogenesis is due to local rather than systemic immunosuppression. Cytokine production from these EBV-immortalized cells can build up to high levels in enclosed spaces. Increase in interleukin-10, which inhibits $\mathrm{T}$ cell proliferation due to inhibition of antigen presenting cells, may contribute to this local
Fig. 6 Further workup done on the amputation specimen showing in situ hybridization studies for EBER (a), and in situ hybridization studies for kappa (b) and lambda (c), showing a kappa light chain restriction
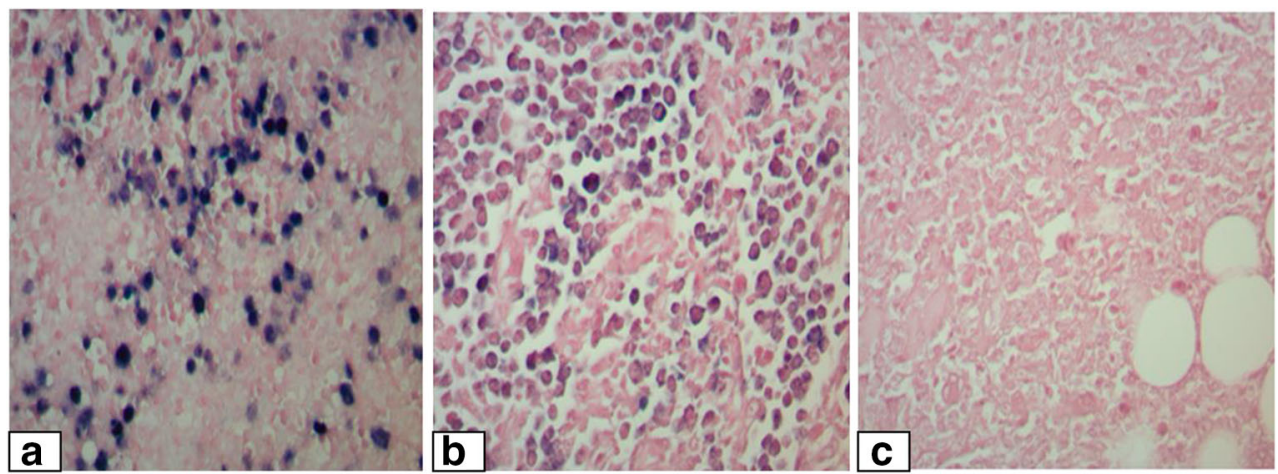
Fig. 7 Immunohistochemical markers of immune checkpoints, showing positivity for PDL1 and TIM3, and negativity for PD1 and LAG3
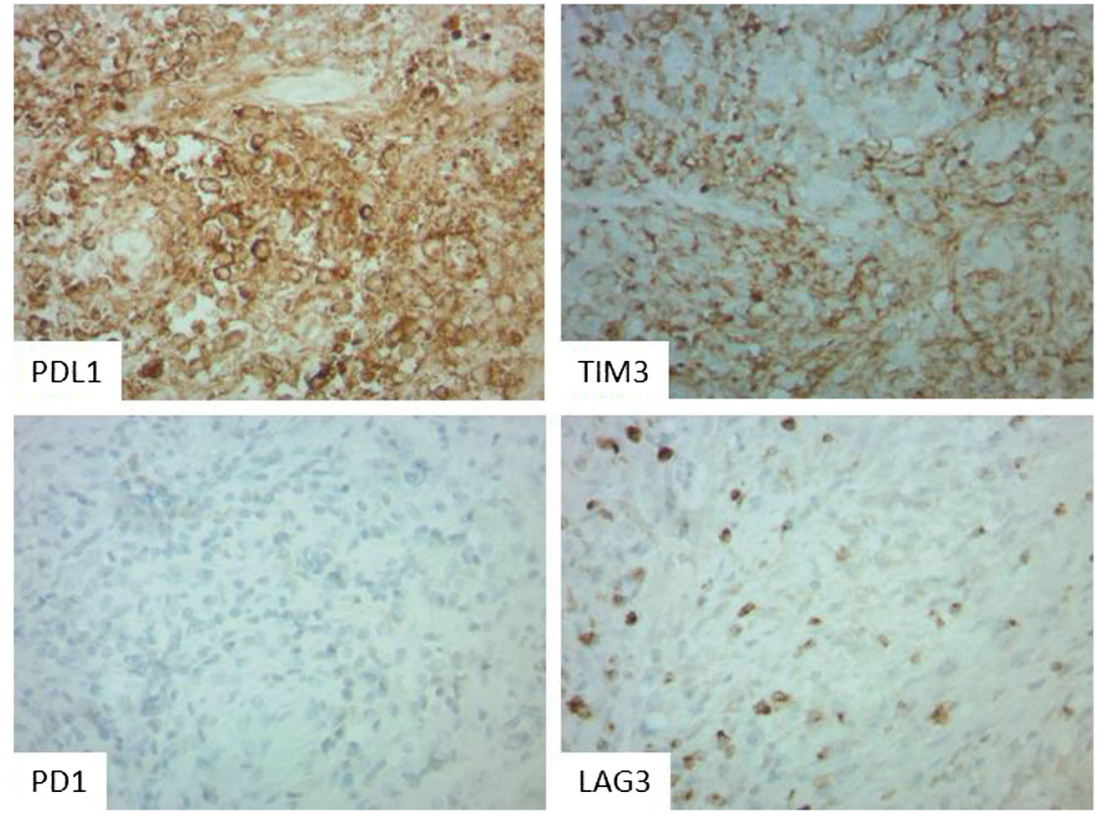

immunosuppression [6]. A previous case series published in 1997 reported three cases of non-Hodgkin lymphomas associated with long-standing chronic suppuration. Two of these three cases demonstrated morphology and immunophenotype consistent with PBL [11]. Additionally, ineffective T cell response with expression of programmed cell death ligand 1 (PD-L1) on tumor cells and macrophages has been described in aggressive $\mathrm{B}$ cell malignancies and virus-associated malignancies [12]. PD-L1 is a cell-surface glycoprotein expressed by antigen-presenting cells to regulate the cellular immune response [13]. Binding of PD-L1 to its receptor PD-1 inhibits proliferation of activated $\mathrm{T}$ cells resulting in immune modulation [14]. We believe that this could be the pathogenesis behind the development of PBL in our case. Recently, there has been a significant advance in the treatment of hematologic malignancies, including lymphoma, using anti-PD-1 and anti-PD-L1 immunotherapeutic agents. PD-L1 protein expression has been described in certain aggressive B cell lymphomas and virus- and immunodeficiency-associated malignancies associated with an ineffective $\mathrm{T}$ cell immune, one of which is PBL [12]. Signaling through additional immune checkpoint receptors, such as lymphocyte-activation gene-3 (LAG-3) and T cell immunoglobulin and mucin domain-3 (TIM-3), that leads to $\mathrm{T}$ cell exhaustion has been recently described [15]. In the current case, immunohistochemical staining showed positivity for PDL1 and TIM3 and negative staining for LAG3 and PD1.

Our case is an unusual presentation of PBL. Typically, the clinical scenario involves an immunocompromised patient with an oral lesion. Soft tissue lesions are uncommon. Our patient was immunocompetent with a soft tissue lesion in the left calcaneal area. Serum protein electrophoresis showed no abnormalities, and imaging studies showed no abnormality in bone density. The initial biopsy was also misleading, probably due to the degree of necrosis found in the ulcerated areas. The differential diagnosis included a metastatic carcinoma and high-grade sarcoma. Due to the nature and location of the lesion, surgical management was pursued resulting in an amputation for local control.

Localized chronic inflammation may have contributed to the pathogenesis of this neoplasm. EBER in situ hybridization diffuse positivity and PDL1 expression support this diagnosis. This was a difficult pathologic diagnosis given that the lesion was negative for CD45. Clinical history and ancillary studies including EBER and PDL1 helped elucidate the final diagnosis.

Following the surgery, the patient was lost to follow-up for 8 months. When he presented again for clinical evaluation, he was found to have significant swelling of the left thigh above the amputation site as well as in the left inguinal area. He also complained of excruciating pain, weight loss, and fatigue. Follow-up PET scan, at that time, revealed large hypermetabolic soft tissue lesions in the left lower abdomen, the proximal left thigh and leg, left inguinal and external iliac lymph nodes, and perhaps within the small bowel, consistent with disease progression. Fine needle aspiration of the left thigh soft tissue mass was performed, which was confirmed to be PBL on cytologic evaluation and immunohistochemistry performed on the cell block (Fig. 8). Cytokeratin AE1/AE3 was negative. There was focal immunoreactivity for CD38 and CD79a, while CD138 showed rare positive. EBER ISH was positive in tumor cells. MUM1 was focally positive. 
Fig. 8 Cytology from the fine needle aspirate specimen shows discohesive cells with immunoblastic and plasmacytoid features (a and $\mathbf{b}$, high power views). There was focal immunoreactivity for CD38 (c) and CD79a (d), while CD138 (e) showed rare positive. EBER ISH (f) was positive in tumor cells. MUM1 was focally positive

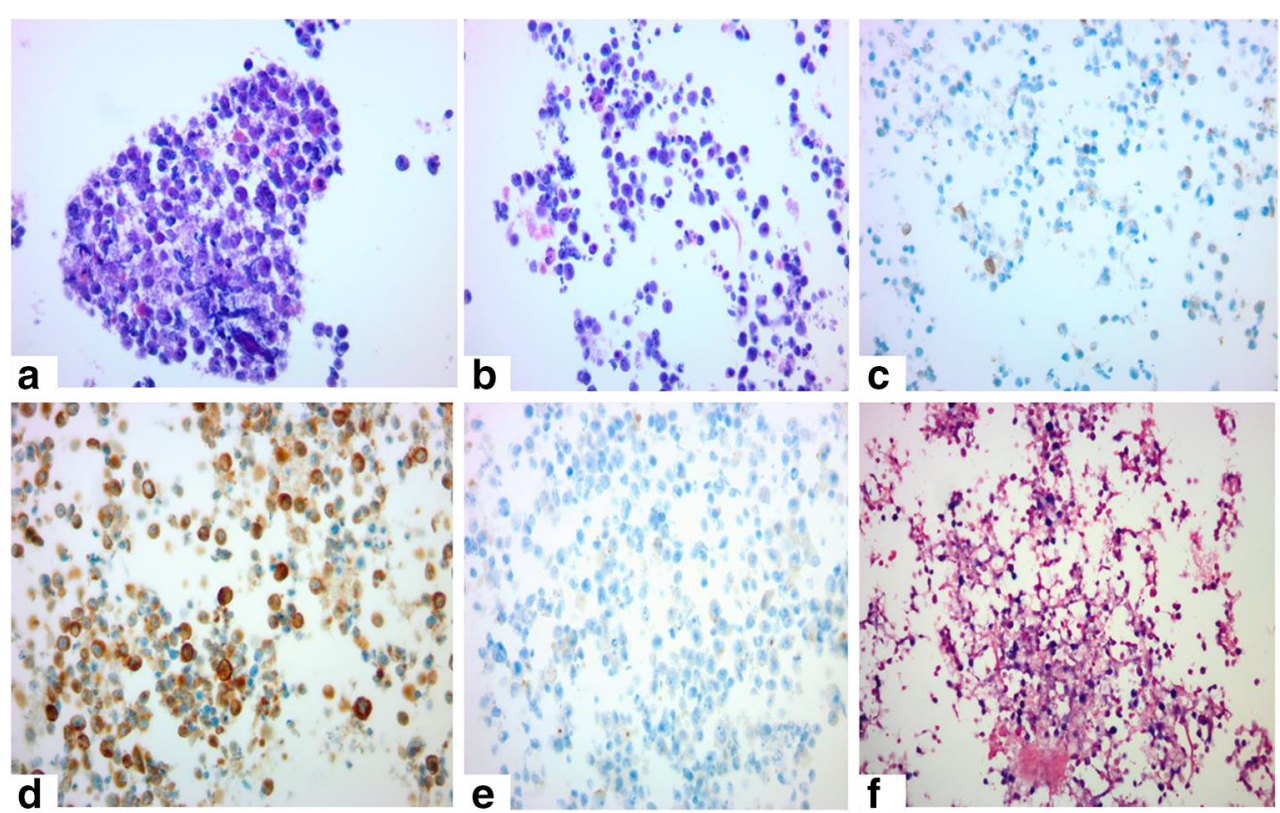

Consequently, the patient was treated with 5 cycles of CHOP chemotherapy with bortezomib, which includes cyclophosphamide $750 \mathrm{mg} / \mathrm{m}^{2}$, adriamycin $50 \mathrm{mg} / \mathrm{m}^{2}$, and vincristine $2 \mathrm{mg}$ intravenously as well as prednisone $80 \mathrm{mg}$ daily. $\mathrm{He}$ completed 5 cycles but declined the last cycle because of intolerance to side effects. He also received emergency radiation therapy to the left thigh with good results. He was placed on maintenance lenalidomide $10 \mathrm{mg}$ daily, which was discontinued due to side effects intolerance. Currently, the patient is feeling well and is using prosthesis without any assistive devices to walk. He is independent in all his activities of daily living.

\section{Conclusion}

PBL is an aggressive lymphoma with a predilection for the head and neck, particularly the oral cavity, usually in immunocompromised patients. Presentation in rare locations may pose a diagnostic dilemma, given that CD45, CD20, and PAX5 are usually either negative or weakly positive, in addition to extensive necrosis which may be present in some cases, precluding accurate interpretation of immunoperoxidase stains on a limited biopsy specimen. Despite being rare, PBL should be included in the differential diagnostic workup of soft tissue tumors with uncertain line of differentiation, especially if it shows an anaplastic or pleomorphic cytomorphology. Plasma cell markers such as CD138, CD38, as well as CD79a and MUM1, EBER, and PDL1 may be helpful stains in confirming the diagnosis.

\section{Compliance with ethical standards}

Conflict of interest The authors declare that they have no conflict of interest.

\section{References}

1. Delecluse HJ, Anagnostopoulos I, Dallenbach F, Hummel M, Marafioti T, Schndeider U et al (1997) Plasmablastic lymphomas of the oral cavity: a new entity associated with the human immunodeficiency virus infection. Blood. 89:1413-1420

2. Morscio J, Dierickx D, Nijs J, Verhoef G, Bittoun E, Vanoeteren X, Wlodarska I, Sagaert X, Tousseyn T (2014) Clinicopathologic comparison of plasmablastic lymphoma in HIV-positive, immunocompetent and posttransplant patients. Am J Surg Pathol 38:875-886

3. Loghavi S, Alayed K, Aladily TN, Zuo Z, Ng SB, Tang G, Hu S, Yin CC, Miranda RN, Medeiros LJ, Khoury JD (2015) Stage, age, and EBV status impact outcomes of plasmablastic lymphoma patients: a clinicopathologic analysis of 61 patients. J Hematol Oncol $8: 65$

4. Segal GH, Jorgensen T, Scott M, Braylan RC (1994) Optimal primer selection for clonality assessment by polymerase chain reaction analysis: II. Follicular lymphomas. Hum Pathol 25(12):1276-1282

5. Castillo JJ, Bibas M, Miranda N (2015) The biology and treatment of plasmablastic lymphoma. Blood. 125:2323-2330

6. Loong F et al (2016) Diffuse large B-cell lymphoma associated with chronic inflammation as an incidental finding and new clinical scenarios. Mod Pathol 23:493-501

7. Ahn JS, Okal R, Vos JA, Smoklin M, Kanate AS, Rosado FG (2017) Plasmablastic lymphoma versus plasmablastic myeloma: an ongoing diagnostic dilemma. J Clin Pathol 0:1-6

8. Vega F, Chang CC, Medeiros LJ, Udden MM, Cho-Vega JH, Lau CC, Finch CJ, Vilchez RA, McGregor D, Jorgensen JL (2005) Plasmablastic lymphomas and plasmablastic plasma cell myelomas have nearly identical immunophenotypic profiles. Mod Pathol 18: 806-815 
9. Harmon CM, Smith LB (2016) Plasmablastic lymphoma: a review of clinicopathologic features and differential diagnosis. Arch pathol Lab med 140:1074-1078

10. Dongen V et al (2003) Design and standardization of PCR primers and protocols for detection of clonal immunoglobulin and T-cell receptor gene recombinations in suspect lymphoproliferations: report of the BIOMED-2 Concerted Action BMH4-CT98-3936. Leukemia 17:2257-2317

11. Copie-bergman C et al (1997) Epstein-Barr virus in B-cell lymphomas associated with chronic suppurative inflammation. J Pathol 183:287-292

12. Chen BJ, Chapuy B, Ouyang J, Sun HH, Roemer MGM, Xu ML, Yu H, Fletcher CDM, Freeman GJ, Shipp MA, Rodig SJ (2013) PD-L1 expression is characteristic of a subset of aggressive B-cell lymphomas and virus-associated malignancies. Clin Cancer Res 19(13):3462-3473
13. Keir ME, Butte MJ, Freeman GJ, Sharpe AH (2008) PD-1 and its ligands in tolerance and immunity. Annu Rev Immunol 26:677-704

14. Freeman GJ, Long AJ, Iwai Y, Bourque K, Chernova T, Nishimura H, Fitz LJ, Malenkovich N, Okazaki T, Byrne MC, Horton HF, Fouser L, Carter L, Ling V, Bowman MR, Carreno BM, Collins M, Wood CR, Honjo T (2000) Engagement of the PD-1 immunoinhibitory receptor by a novel B7 family member leads to negative regulation of lymphocyte activation. J Exp Med 192: $1027-1034$

15. Chen BJ, Dashnamoorthy R, Galera P, Makarenko V, Chang H, Ghosh S, Evens AM (2019) The immune checkpoint molecules PD-1, PD-L1, TIM-3 and LAG-3 in diffuse large B-cell lymphoma. Oncotarget. 10(21):2030-2040

Publisher's note Springer Nature remains neutral with regard to jurisdictional claims in published maps and institutional affiliations. 